\title{
Association between telomere length and complete blood count in US adults
}

\author{
Mohsen Mazidi'1,2, Peter Penson ${ }^{3}$, Maciej Banach ${ }^{4,5}$
}

\begin{abstract}
${ }^{1}$ Key State Laboratory of Molecular Developmental Biology, Institute of Genetics and Developmental Biology, Chinese Academy of Sciences, Beijing, China ${ }^{2}$ Institute of Genetics and Developmental Biology, International College, University of Chinese Academy of Science, Beijing, China

${ }^{3}$ School of Pharmacy and Biomolecular Sciences, Liverpool John Moores University, Liverpool, UK

${ }^{4}$ Department of Hypertension, Chair of Nephrology and Hypertension, Medical University of Lodz, Lodz, Poland

${ }^{5}$ Cardiovascular Research Centre, University of Zielona Gora, Zielona Gora, Poland
\end{abstract}

Submitted: 2 February 2017

Accepted: 10 February 2017

Arch Med Sci 2017; 13, 3: 601-605

DOI: https://doi.org/10.5114/aoms.2017.67281

Copyright $\odot 2017$ Termedia \& Banach

\section{Abstract}

Introduction: Telomere length $(\mathrm{TL})$ is related to age-related health outcomes, but little is known about the relationship between TL and complete blood count $(C B C)$ parameters. We aimed to determine the relationship between $\mathrm{TL}$ and $C B C$ in a sample of healthy US adults.

Material and methods: Participants in the National Health and Nutrition Examination Survey (NHANES) recruited between 1999 and 2002 who had essential data on total CBC and TL were studied. We computed ageand race-adjusted mean values for total $C B C$ using analysis of covariance (ANCOVA). All statistical analyses accounted for the survey design and sample weights by using SPSS Complex Samples v22.0 (IBM Corp, Armonk, NY). Results: Of the 8892 eligible participants, $47.8 \%(n=4123)$ were men. The mean age was 41.8 years overall, 41.0 years in men and 42.6 in women $(p=0.238)$. The sex-stratified ANCOVA showed no significant difference in the total CBC across TL quartiles (all $p>0.05$ ) in both sexes. In the adjusted model, there was a significant negative relationship with monocyte count $(\beta=-0.051,95 \% \mathrm{Cl}:-0.422 ;-0.142)$, mean cell hemoglobin $(\beta=-0.051$, $95 \% \mathrm{Cl}:-0.038 ;-0.011)$ and red cell distribution width $(\beta=-0.031,95 \% \mathrm{Cl}$ : $-0.054 ;-0.003)$, while there was a significant positive relationship with basophil ratio $(\beta=0.046,95 \% \mathrm{Cl}: 0.049-0.171)$.

Conclusions: These results support the possibility that telomere attrition may be a marker for reduced proliferative reserve in hematopoietic progenitor cells.

Key words: telomere length, complete blood count, National Health and Nutrition Examination Survey.

\section{Introduction}

Telomeres are nucleotide sequences located at the ends of chromosomes that assist in the preservation of chromosomal mechanical reliability and genomic constancy [1]. It has been reported that telomere length ( $\mathrm{TL}$ ) of replicating somatic cells is inversely associated with age and age-related disorders, such as cardiovascular diseases (CVDs) [1, 2]. The development of bone marrow aging includes slow replacement of he-

\author{
Corresponding author: \\ Mohsen Mazidi \\ Key State Laboratory \\ of Molecular \\ Developmental Biology \\ Institute of Genetics \\ and Developmental Biology \\ Chinese Academy \\ of Sciences \\ Beijing 100101, China \\ E-mail: Moshen@genetics. \\ ac.cn
}


matopoietic tissue by adipocytes and fibroblasts [3]. This process is mainly observed in subjects more than 80 years old, who have $50 \%$ less hematopoietic tissue than younger subjects [4].

The bone marrow aging process is also associated with increased DNA impairment in hematopoietic stem cells (HSCs) [5]. Although it has been stated that telomerase activity in HSCS would preserve a constant TL [6], recently, it has been reported that telomere shortening can restrict the preservation and functions of the adult stem cells [7]. It is possible that the telomerase activity in HSCs is insufficient to avert telomere destruction and, consequently, unable to avert cell senescence [8]. It has been reported that the telomerase activity varies in HSCs from very weak to a level of activity similar to that seen in immortal cell lines, with no age-dependent deterioration [9]. To date, the relationship between TL and peripheral blood count factors has been explored in middle-aged [10], combined middle-aged and elderly [11], and elderly [12] subjects. However, the findings of these studies are controversial. Hence, it is still largely unknown how telomere maintenance might be associated with blood count parameters. In this regard, we aimed to determine the association between TL and blood count parameters in healthy adults from the National Health and Nutrition Examination Surveys (NHANES) cohort.

\section{Material and methods}

\section{Population}

National Health and Nutrition Examination Surveys are ongoing repeated cross sectional surveys conducted by the US National Center for Health Statistics (NCHS) [2, 13-15]. The NHANES uses a multistage probability sampling strategy, which oversamples certain subgroups of the population, including blacks, Mexican-Americans, and those of lower socioeconomic status $[2,13,14,16]$. The National Center for Health Statistics Research Ethics Review Board approved the NHANES protocol, and consent was obtained from all participants (informed consent was obtained from all subjects) $[2,13,17]$. Moreover, all methods were performed in accordance with the relevant guidelines and regulations [2, 13, 14]. Data collection on demographic information is conducted through in-home administered questionnaires, while anthropometric, inflammatory and biochemical data are collected by trained persons using mobile examination units. More detailed information on the NHANES is available elsewhere $[13,18]$. The current study was based on analysis of data for two 2-year NHANES survey cycles: 1999-2000 and 2001-2002. Adults with a history of diabetes, coronary heart disease, angina, myocardial infarction, stroke or congestive heart failure were excluded.
We restricted our analysis to subjects more than 18 years old. The main response variables of interest were 13 types of cellular blood composition: hemoglobin (HGB), mean cell hemoglobin $(\mathrm{MCH})$, mean cell volume (MCV), hematocrit (HCT), red blood cell count (RBC), platelet count (PLT), basophil number (BAS), eosinophil number (EOS), monocyte number (Mon), segmented neutrophil number (Neu), lymphocyte number (Lym), neutrophil/lymphocyte ratio (NLR), and white blood cell count (WBC). The CBC analysis was performed using the Coulter method, following the NHANES quality control and quality assurance protocols [19].

\section{Leukocyte telomere length measurements}

Briefly, aliquots of purified DNA, isolated from whole blood using the Puregene (D-50 K) kit protocol (Gentra Systems, Inc., Minneapolis, Minnesota), were obtained from participants. The leukocyte TL assay was performed using the quantitative polymerase chain reaction method to measure TL relative to standard reference DNA (also known as the telomere-to-single (T/S) ratio) [20]. Each sample was assayed 3 times on 3 different days. The samples were assayed on duplicate wells, resulting in 6 data points. Control DNA values were used to normalize between-run variability. The Centers for Diseases Control (CDC) conducted a quality control review before linking the TL data to the NHANES data files. The CDC Institutional Review Board granted human subject approval for this study $[2,17]$.

\section{Statistical analysis}

We conducted the analyses according to the guidelines set out by the CDS for analysis of the complex NHANES dataset, accounting for the masked variance and using the proposed weighting methodology $[2,16,21]$. We computed ageand race-adjusted values of $C B C$ by using analysis of covariance (ANCOVA). Categorical demographic variables were compared by using analysis of variance (ANOVA) and the $\chi^{2}$ test. Fully adjusted multivariate linear regression (stepwise mode) and partial correlation were used to determine the association of CBC by TL. All tests were two sided, and $p<0.05$ was the level of significance. Results were analyzed using SPSS complex sample module version 22.0 (IBM Corp, Armonk, NY). Sample weights were applied to account for unequal probabilities of selection, nonresponse bias, and oversampling.

\section{Results}

The weighted distributions of study population characteristics are shown in Table I. Of the 8892 eligible participants, $47.8 \%(n=4123)$ were men. The mean age of the total population sample was 
41.8 years, 41.0 years in men and 42.6 in women $(p=0.238)$. It can be seen in Table II that whites (non-Hispanic) comprised the majority (70.3\%) of the population in the study. Moreover, 55.5\% $(n=4294)$ of the sample consisted of married subjects. The mean and SEM for the TL in the total population was $1.08 \pm 0.015$, and for males and females it was $1.07 \pm 0.014$ and $1.08 \pm 0.016$, respectively.

In the sex-stratified ANCOVA (age and race adjusted), we found no significant difference in the values of the parameters comprising the CBC across TL quartiles (all $p>0.05$ ) in either sex. In the adjusted model, we found a significant negative relationship of $\mathrm{TL}$ with monocyte count ( $\beta=-0.051,95 \% \mathrm{Cl}:-0.422 ;-0.142)$, mean cell hemoglobin ( $\beta=-0.051,95 \% \mathrm{Cl}$ : $-0.038 ;-0.011)$ and red cell distribution width $(\beta=-0.031,95 \%$ $\mathrm{Cl}$ : -0.054 ; -0.003$)$, while there was a significant positive relationship with basophil ratio $(\beta=0.046,95 \% \mathrm{Cl}: 0.049-0.171)$ (Table II).

\section{Discussion}

This study investigated the relationship between telomere length and complete blood count in healthy US adults. We found a positive associa- tion between $T L$ and the percentage of basophils, but a significant negative relationship between $\mathrm{TL}$ and monocyte count, mean cell hemoglobin and red cell distribution width. The association between telomere length and complete blood count has been reported in the literature [22-24]. Compte et al. found that telomere length was associated with total WBC count and neutrophils and CV diseases with monocytes. Moreover, the findings of a national investigation in the US revealed an inverse association between MCV and telomere length in a large urban US population, and suggested a biologic mechanism for macrocytosis of aging [23].

In contrast with our findings, De Meyer et al. found a positive association of TL and red blood cell count but no significant association of TL and hematocrit, hemoglobin or leukocytes in middle-aged subjects in the Asklepios study [25]. Interestingly, some of the investigations found no significant association between telomere length and complete blood count in elderly individuals $[26,27]$. Den Elzen et al. proposed an explanation for the lack of association between telomere length and hematological parameters in elderly people - that telomere length in WBC in old age

Table I. Sample size and weighted characteristics of NHANES 1999-2002 adult participants

\begin{tabular}{|lllc|}
\hline Parameter & \multicolumn{1}{c}{$\boldsymbol{N}$} & Weighted distributions of the participants \\
\hline Gender & Men & 4123 & $47.8 \%$ \\
\cline { 2 - 4 } & Women & 4769 & $52.6 \%$ \\
\hline Age, mean \pm SEM [years] & 8892 & $41.82 \pm 0.28$ \\
\hline Education level & Less than high school & 2461 & $19.8 \%$ \\
\cline { 2 - 4 } & Completed high school & 1815 & $25.4 \%$ \\
\cline { 2 - 4 } & More than high school & 3507 & $54.6 \%$ \\
\hline Race/ethnicity & White (non-Hispanic) & 4066 & $70.3 \%$ \\
\cline { 2 - 4 } & Non-Hispanic black & 1738 & $10.7 \%$ \\
\cline { 2 - 4 } & Mexican-American & 2315 & $7.2 \%$ \\
\cline { 2 - 4 } & Other Hispanic and other & 773 & $11.8 \%$ \\
\hline
\end{tabular}

Table II. Multivariate linear regression (stepwise mode) and partial correlation (100 bootstrap replications) for determining the relation between telomere length and $C B C$

\begin{tabular}{|lccc|}
\hline Variables & \multicolumn{2}{c|}{ Linear regression } & Partial correlation \\
\cline { 2 - 4 } & $\beta$ coefficient & $95 \% \mathrm{Cl}$ & Coefficient \\
\hline Monocyte count & -0.051 & $(-0.422,-0.142)$ & $-0.057^{*}$ \\
\hline Basophils (\%) & 0.046 & $(0.049-0.171)$ & $0.048^{*}$ \\
\hline Mean cell hemoglobin [pg] & -0.051 & $(-0.038,-0.011)$ & $-0.43^{*}$ \\
\hline Red cell distribution width (\%) & -0.031 & $(-0.054,-0.003)$ & -0.08 \\
\hline
\end{tabular}

Both models adjusted for age, race, gender, and body mass index. ${ }^{*} p>0.001$. 
is unstable and does not have enough predictive power for age-related morbidity and mortality at ages over 85 years $[26,28]$. However, Gutmajster et al. found a weak and independent correlation of TL with both RBC and WBC in elderly individuals [29]. Therefore, the type of cells studied (peripheral blood mononuclear cells, hematopoietic cells, lymphocytes, etc.) and differences in studied groups should be considered when interpreting clinical studies and trials.

Interestingly, some of the recent literature stated that telomere length varies according to the type of lymphocytes studied: B cells have the longest telomere length and highest telomerase activity, in contrast to CD8+ CD28- T cells; moreover, women have longer telomeres than men and may be protected by their hormonal status [22, 30]. Shorter TL in men may explain gender-specific dissimilarities in the degree of peripheral blood cell count. Therefore, in comparison to women, it should be easier to identify associations in men [29]. In addition, other potential confounders in cluding stress and cortisol can affect telomere length and WBC counts [22, 30, 31].

This study has several strengths. To our knowledge, it is the largest study of the association of CBC and TL. The study is sufficiently powered to test the associations. The selection of the participants was based on random sampling of the general population, and therefore the results obtained from nationally representative samples can be extrapolated to the general population.

The findings from our study have to be considered in the context of some study limitations. The cross-sectional nature does not allow inference about causality. Unfortunately, we did not have available any repeated measure of TL with quantitative polymerase chain reaction in the same subjects after several follow-up years to elucidate the temporality of these findings.

This study has important clinical and public health implications. Understanding the interplay between CBC and TL is a necessary and important step toward any application of the resulting knowledge for public health policy and action, as it has been suggested that shorter telomeres have been associated with coronary heart disease [32, 33]. Our study provides a comprehensive snapshot of the relationship of CBC with TL at the national level in the US.

In conclusion, according to the findings of the present study, it can be concluded that telomere length is associated with blood cell parameters. Further investigations with a rigorous study design allowing correction for factors known to influence telomere length are necessary in order to clarify the clinical implications of measuring this parameter.

\section{Acknowledgments}

Mohsen Mazidi was supported by a TWAS studentship of the Chinese Academy of Sciences, during the preparation of this manuscript.

\section{Conflict of interest}

The authors declare no conflict of interest.

\section{References}

1. Aviv A. Telomeres and human aging: facts and fibs. Sci Aging Knowledge Environ 2004; 2004: pe43.

2. Mazidi M, Michos ED, Banach M. The association of telomere length and serum 25-hydroxyvitamin D levels in US adults: the National Health and Nutrition Examination Survey. Arch Med Sci 2017; 13: 61-5.

3. Hirota Y, Okamura S, Kimura N, Shibuya T, Niho Y. Haematopoiesis in the aged as studied by in vitro colony assay. Eur J Haematol 1988; 40: 83-90.

4. Ogawa T, Kitagawa M, Hirokawa K. Age-related changes of human bone marrow: a histometric estimation of proliferative cells, apoptotic cells, T cells, B cells and macrophages. Mech Ageing Dev 2000; 117: 57-68.

5. Rube CE, Fricke A, Widmann TA, et al. Accumulation of DNA damage in hematopoietic stem and progenitor cells during human aging. PLoS One 2011; 6: e17487.

6. Weng N. Interplay between telomere length and telomerase in human leukocyte differentiation and aging. J Leukoc Biol 2001; 70: 861-7.

7. Nalapareddy K, Jiang H, Guachalla Gutierrez LM, Rudolph KL. Determining the influence of telomere dysfunction and DNA damage on stem and progenitor cell aging: what markers can we use? Exp Gerontol 2008; 43: 998-1004.

8. Engelhardt M, Kumar R, Albanell J, Pettengell R, Han W, Moore MA. Telomerase regulation, cell cycle, and telomere stability in primitive hematopoietic cells. Blood 1997; 90: 182-93.

9. Wang JC, Warner JK, Erdmann N, Lansdorp PM, Harrington L, Dick JE. Dissociation of telomerase activity and telomere length maintenance in primitive human hematopoietic cells. Proc Natl Acad Sci U S A 2005; 102: 14398-403.

10. De Meyer T, De Buyzere ML, Langlois M, et al. Lower red blood cell counts in middle-aged subjects with shorter peripheral blood leukocyte telomere length. Aging Cell 2008; 7: 700-5.

11. Mollica L, Fleury I, Belisle C, Provost S, Roy DC, Busque L. No association between telomere length and blood cell counts in elderly individuals. J Gerontol A Biol Sci Med Sci 2009; 64: 965-7.

12. Den Elzen WP, Martin-Ruiz C, von Zglinicki T, Westendorp RG, Kirkwood TB, Gussekloo J. Telomere length and anaemia in old age: results from the Newcastle 85-plus Study and the Leiden 85-plus Study. Age Ageing 2011; 40: 494-500.

13. National Center for Health Statistics. National Health and NutritionExamination Survey (NHANES). Questionnaires, datasets, and related documentation. www. cdc.gov/nchs/nhanes/nhanes_questionnaires.htm. Accessed January 27, 2015.

14. Mazidi M, Gao HK, Vatanparast H, Kengne AP. Impact of the dietary fatty acid intake on C-reactive protein levels in US adults. Medicine (Baltimore) 2017; 96: e5736. 
15. Mazidi M, Pennathur S, Afshinnia F. Link of dietary patterns with metabolic syndrome: analysis of the National Health and Nutrition Examination Survey. Nutr Diabetes 2017; 7: e255.

16. Mazidi M, Kengne AP. Nutrient patterns and their relationship with general and central obesity in US adults. Eur J Clin Invest 2017 Mar 10. doi: 10.1111/eci.12745.

17. Mazidi M, Kengne AP, Banach M. Mineral and vitamin consumption and telomere length among adults in the United States. Pol Arch Intern Med 2017; 127: 87-90.

18. Mazidi M, Gao HK, Vatanparast H, Kengne AP. Impact of the dietary fatty acid intake on C-reactive protein levels in US adults. Medicine (Baltimore) 2017; 96: e5736.

19. Zipf G, Chiappa M, Porter KS, Ostchega Y, Lewis BG, Dostal J. National health and nutrition examination survey: plan and operations, 1999-2010. Vital Health Stat 1 2013; 56: 1-37.

20. Needham BL, Adler N, Gregorich S, et al. Socioeconomic status, health behavior, and leukocyte telomere length in the National Health and Nutrition Examination Survey, 1999-2002. Soc Sci Med 2013; 85: 1-8.

21. Statistics. NCFH. ANALYTIC AND REPORTING GUIDELINES http://www.cdc.gov/nchs/data/nhanes/ nhanes 03 04/nhanes analytic guidelines dec 2005.pdf.

22. Compte N, Bailly B, De Breucker S, Goriely S, Pepersack T. Study of the association of total and differential white blood cell counts with geriatric conditions, cardio-vascular diseases, seric IL-6 levels and telomere length. Exp Gerontol 2015; 61: 105-12.

23. Kozlitina J, Garcia CK. Red blood cell size is inversely associated with leukocyte telomere length in a large multi-ethnic population. PLoS One 2012; 7: e51046.

24. Neuner B, Lenfers A, Kelsch R, et al. Telomere length is not related to established cardiovascular risk factors but does correlate with red and white blood cell counts in a German blood donor population. PLoS One 2015; 10: e0139308.

25. De Meyer T, De Buyzere ML, Denil S, et al. No shorter telomeres in subjects with a family history of cardiovascular disease in the Asklepios study. Arterioscler Thromb Vasc Biol 2012; 32: 3076-81.

26. Den Elzen WP, Martin-Ruiz C, von Zglinicki T, Westendorp RG, Kirkwood TB, Gussekloo J. Telomere length and anaemia in old age: results from the Newcastle 85-plus Study and the Leiden 85-plus Study. Age Ageing 2011; 40: 494-500.

27. Mollica L, Fleury I, Belisle C, Provost S, Roy DC, Busque L No association between telomere length and blood cell counts in elderly individuals. J Gerontol A Biol Sci Med Sci 2009; 64: 965-7.

28. Martin-Ruiz CM, Gussekloo J, Heemst D, Zglinicki T, Westendorp RG. Telomere length in white blood cells is not associated with morbidity or mortality in the oldest old: a population-based study. Aging Cell 2005; 4: 287-90.

29. Gutmajster E, Witecka J, Wyskida M, et al. Telomere length in elderly Caucasians weakly correlates with blood cell counts. Sci World J 2013; 2013: 153608.

30. Andrews NP, Fujii H, Goronzy JJ, Weyand CM. Telomeres and immunological diseases of aging. Gerontology 2009; 56: 390-403.

31. Lin J, Epel E, Cheon J, et al. Analyses and comparisons of telomerase activity and telomere length in human $T$ and $B$ cells: insights for epidemiology of telomere maintenance. J Immunol Methods 2010; 352: 71-80.

32. Huzen J, Peeters W, de Boer RA, et al. Circulating leukocyte and carotid atherosclerotic plaque telomere length interrelation, association with plaque characteristics, and restenosis after endarterectomy. Arterioscler Thromb Vasc Biol 2011; 31: 1219-25.

33. Wilson WRW, Herbert KE, Mistry Y, et al. Blood leucocyte telomere DNA content predicts vascular telomere DNA content in humans with and without vascular disease. Eur Heart J 2008; 29: 2689-94. 